\title{
Reply to 'Comment on 'Human papillomavirus association is the most important predictor for surgically treated patients with oropharyngeal cancer"
}

Steffen Wagner ${ }^{1,8}$, Claus Wittekindt ${ }^{1,8}$, Shachi Jenny Sharma ${ }^{1}$, Nora Wuerdemann ${ }^{1}$, Theresa Jüttner ${ }^{1}$, Miriam Reuschenbach $^{2,3}$, Elena-Sophie Prigge ${ }^{2,3}$, Magnus von Knebel Doeberitz ${ }^{2,3}$, Stefan Gattenlöhner ${ }^{4}$, Ernst Burkhardtt ${ }^{5,6,7}$, Jörn Pons-Kühnemann ${ }^{5,9^{\prime}}$ and Jens Peter Klussmann ${ }^{\star 1,9}$

\begin{abstract}
${ }^{1}$ Department of Otorhinolaryngology, Head and Neck Surgery, University of Giessen, Giessen DE-35392, Germany; ${ }^{2}$ Department of Applied Tumour Biology, Institute of Pathology, University Hospital Heidelberg, Heidelberg D-69120, Germany; ${ }^{3}$ Clinical Cooperation Unit Applied Tumour Biology, German Cancer Research Centre (DKFZ), Heidelberg D-69120, Germany; ${ }^{4}$ Department of Pathology, University of Giessen, Giessen DE35392, Germany; ${ }^{5}$ Department of Medical Statistics, Institute of Medical Informatics, University of Giessen, Giessen DE-35392, Germany; ${ }^{6}$ Tumour Centre, University of Giessen, Giessen DE-35392, Germany and ${ }^{7}$ Hesse Cancer Registry, Frankfurt DE-60439, Germany
\end{abstract}

Sir,

We thank Bossi et al (2018) for their valuable comments on our manuscript 'Human papillomavirus (HPV) association is the most important predictor for surgically treated patients with oropharyngeal cancer' (Wagner et al, 2017). Concerning their remarks, we would like to address the following clarification.

Bossi et al (2018) criticise a bias according the selection of therapy. However, we included all patients in the period independent of treatment, stage, age or performance status. For the risk model generation by recursive partitioning analysis (RPA), only patients treated without curative intent were excluded. Most of our patients received upfront surgery, which is a therapeutic option following the guidelines for oropharyngeal squamous cell carcinoma (OPSCC), and treatment strategies in this retrospective study are not balanced. However, it was exactly in our focus to create a risk model for patients that primarily receive surgery for treatment of OPSCC. The need for such a model is unquestionable because many patients with cancer of the oropharynx receive upfront surgery. Surgery was the predominant treatment in the period and did not change over time. The cohort is unselected because all consecutive patients were included in our model in order to depict 'reallife'. Most importantly, the selection of treatment, neither guided to prefer surgery nor not, did not influence our analysis since treatment was not used for risk model generation. The overall survival of the surgical group is better than that of the non-surgical group, and this can result from selection. Nevertheless, the aim our study was not to compare surgical vs non-surgical treatment, and therefore we compared survival regarding treatment within the resulting (RPA-based) risk groups only (Figure 3 of Wagner et al, 2017).

Another concern was the quality of the data regarding smoking and performance. We acknowledge that superior measurements of patientrelated risk factors exist, for example, applicable in clinical trials. Our data were from standardised sheets in patient's charts and categorised to best means. A 10-pack-year cutoff in accordance with previous risk models seemed to be adequately sufficient for risk model generation in our study. We used 'moderate or severe comorbidity graded by the Eastern Cooperative Oncology Group (ECOG) 0-1 vs 2-4' as variable. Since no other index has been used, we think it is clear for readers that only this index has been applied.

A further concern is that patients with all stages (early stages and metastatic disease) amenable for curative therapy were implemented into our study. We want to clarify that patients not amenable for curative therapy were not included into the risk modeling. Twenty-three patients had suspicious oligometastatic lesions of the lung, liver or skeleton that later progressed, and therefore were classified $\mathrm{M}+$. However, treatment was in curative intent, for example by local radiation or partial lung resection. We also want to point out that it was in the focus of our analyses to create a risk model for all patients, irrespective of their tumour stage, since such a model is of particular value for clinicians. In addition, it is unlikely that stage migration by a mix of radiologically and pathologically staged cancers influenced the results of our study, as indicated by Bossi et al. We included TNM-stage and not UICC-stage in our model. Grouping of UICC-stages I-III vs UICC-stage IV produced the most significant separation of patients during univariate survival analysis and this is included in the descriptive data presented in Table 1 , however, UICC-stage was not used for further modeling. Differences between radiological and pathological staging are most likely concerning $\mathrm{N}$-stage, which is of minor importance in our model, while T-stage can be well estimated radiologically. Finally, discrepancies are not restricted to upstaging and non-conformity should have less influence due to dichotomising of the variables, which we applied in our study.

In conclusion, our study has limitations that are typically related to the retrospective design; however, our data clearly show that risk models cannot be employed without considering respective treatment standards. Finally, randomised data comparing treatment strategies in the oropharynx is missing in the literature and only data from retrospective cohorts (like ours) exist until now. Therefore, prospective studies are required like EORTC 1420-HNCG-ROG (https://clinicaltrials.gov/ct2/ show/NCT02984410), comparing radiotherapy to trans-oral surgery.

\section{CONFLICT OF INTEREST}

MvKD was shareholder and member of the supervisory board of $\mathrm{mtm}$ Laboratories, Heidelberg, Germany. The remaining authors declare no conflict of interest.

\section{REFERENCES}

Bossi P, Granata R, Orlandi E, Licitra L (2018) Comment on 'Human papillomavirus association is the most important predictor for surgically treated patients with oropharyngeal cancer'. Br J Cancer; e-pub ahead of print 23 January 2018; doi:10.1038/bjc.2017.416.

Wagner S, Wittekindt C, Sharma SJ, Wuerdemann N, Jüttner T, Reuschenbach M, Prigge ES, von Knebel Doeberitz M, Gattenlöhner S, Burkhardt E, PonsKühnemann J, Klussmann JP (2017) Human papillomavirus association is the most important predictor for surgically treated patients with oropharyngeal cancer. BJC 116: 1604-1611.

This work is published under the standard license to publish agree ment. After 12 months the work will become freely available and the license terms will switch to a Creative Commons Attribution-NonCommercial-Share Alike 4.0 Unported License.

*Correspondence: Dr JP Klussmann; E-mail: jens.p.klussmann@hno.med.uni-giessen.de

Published online 23 January 2018

${ }^{8}$ These authors contributed equally as first authors.

${ }^{9}$ These authors contributed equally as senior authors.

(C) 2018 Cancer Research UK. All rights reserved 0007-0920/18 